\title{
Sulla costruzione esplicita di forme differenziali con assegnati periodi.
}

\author{
Memoria di E. Martinelli (a Genova).
}

Sunto. - Si scrivono esplicitamente forme differenziali di grado $\mathrm{p} \leq 4$ che risolvono il pro. blema esisteneiale di CARTAN-DE RHAM in una varietà topologica chiusa n-dimensionale.

1. È noto che un fondamentale teorema di Cartan-De Rham (") assicusa l'esistenza di forme differenziali di grado $p$, regolari e integrabili in una varietà orientabile chiusa $n$-dimensionale $M_{n}$, con periodi preassegnati arbitra. riamente.

In questo lavoro mi occupo della costruzione di una particolare classe di forme differenziali risolventi il predetto problema esistenziale, le quali sono suscettibili di un' espressione esplicita, allorchè, sia la varietà $M_{n}$, sia i cicli di una base per il gruppo di BETTI $(n-p)$-dimensionale di $M_{n}$, possono rappresentarsi localmente mediante parametrizzazioni biunivoche regolari, dotate di derivate di ordine sufficientemente alto.

Benchè io sviluppi qui in completa generalita gli elementi fondamentali, mi limito per ora a giungere fino in fondo alla costruzione delle forme soltanto per i primi quattro valori del grado $p$ (la dimensione $n$ dell' ambiente essendo comunque grande). Per questi valori di $p$ ne deriva una nuova dimostrazione del teorema eitato.

Il mio punto di partenza è l'introduzione di una metrica riemanniana in $M_{n}$. Pur essendo altrimenti nota la possibilità di ot tenere una metrica in in una variet⿱亠े del tipo considerato, segnalo il metodo diretto di cui mi giovo qui a questo scopo (n. 3), metodo che denomino di «sovrapposizione sfu. mata » per illustrarne così il semplice concetto.

Assegnata una varietà $\Delta_{n-p}$ subordinata ad $M_{n}$, fisso poi l'attenzione sul componente normale a $\Delta_{n-p}$ del parallelismo di LEVI-CIVITA determinato dalla metrica. Tale componente, considerato la prima volta da.H. WEYL, consente il trasporto (infinitesimo) di direzioni normali a $\Delta_{n-p}$; lo dico brevemente parallelismo $W$. Ebbene, in relazione al parallelismo $W$ introduco la nozione invariantiva di forma differenziale "inviluppo» $e$, sulla base di questa nozione, pervengo alla costruzione esplicita di forme differenziali risolventi il problema esistenziale predetto. Tali forme risultano definite in modo invariante, una volta assegnata la metrica di $M_{n}$ e scelto un sistema fondamentale per i cicli $(n-p)$-dimensionali della varietà.

(1) Enunciato da E. Cartan [1] e dimostrato da G. De Rham [5]. 
Osservo infine che, se si applica la costruzione qni indicata al caso della varieta, $M_{2 m}$ immagine dello spazio proiettivo complesso $S_{m}$, sulla quale sia fissata la metrica hermitiana ellittica di STudx, si ottengono proprio le note forme differenziali invarianti di WIRTINGER, che forniscono l'elemento di estensione delle immagini in $M_{z m}$ delle varieta analitiche di $S_{m}$. Quest'af* fermazione è almeno valida nei casi che ho fino ad ora esaminato, secondo è precisato al n. 18 .

Questo rapido cenno sul contenuto della ricerca, mostra che il suo carattere è prevalentemente metrico-differenziale. Il teorema di CARTAN-DE RHAM deve invece considerarsi di natura topologica. Forse non è senza interesse questo nuovo esempio dei profondi rapporti che intercorrono fra questi campi apparentemente lontani.

\section{Richiami e premesse.}

2. La varietà ambiente $M_{n}$ și intende connessa, orientabile e chiusa. Si suppone inoltre $M_{n}$ di classe $u$. Ciò significa che $M_{n}$ è ricopribile con un numero finito di intorni $\mathfrak{J}_{n}$, ciascuno dei quali puo deseriversi biunivocamente mediante $n$ coordinate $x_{1}, \ldots, x_{n}$ variabili entro una ipersfera dello spazio euclideo $n$-dimensionale, con la condizione che tra le coordinate $x_{i}, x_{i}^{\prime}(i=1, \ldots, n)$ di uno stesso punto appartenente all'eventuale zona di sovrapposizione di due intorni diversi $\mathfrak{J}_{n}, \mathscr{J}_{n}^{\prime}$ interceda una trasformazione biunivoca con jacobiano non nullo, rappresentata da funzioni di classe $u$ (ammettenti ciò̀ derivate continue fino all'ordine $u$ ).

Si conosca infine in $M_{n}$ una base per il gruppo di BeTrI $(n-p)$-dimensionale, costituita da $(n-p)$-cicli di classe $u_{n}$ rispetto all' ambiente $M_{n}\left({ }^{2}\right)$. Supponiamo $u, u_{0}$ abbastanza alti affinchè abbiano senso gli enti geometrici e analitici in seguito considerati.

Una forma differenziale esterna $\omega_{p}$, di grado $p$, regolare in $M_{n}$ (cioè con coefficienti univocamente definiti e di classe conveniente, in ogni caso però $\geq 1$ ) si dice integrabile (o chiusa), quando si annulla identicamente il suo differen. ziale esterno $d \omega_{p}$; si dice forma differenziale esatto (o omologa a zero), quando esiste una forma $\theta_{p-1}$, di grado $p-1$, regolare in $M_{n}$, tale che $d \theta_{p-1}=\omega_{p}$. Una forma differenziale esatto è sempre integrabile (teorema di PoINoaré); non viceversa in generale.

Ricordiamo che 1'integrale sopra un ciclo $p$-dimensionale $\Gamma_{p}$ di $M_{n}$, di una forma integrabile $\omega_{p}$, non varia sostitnendo il ciclo con un ciclo omologo (anche con divisione) in $M_{n}$. In particolare quell' integrale è nullo se $\Gamma_{p} \approx 0$

(2) Ciò rappresentabili localmente ponendo le coordinate $x_{i}$ di un punto di $M_{n}$ funzioni di elasse $u_{0}$ di $n \quad p$ parametri, con condizioni analoghe a quelle già espresse in relazione ad $M_{n}$ per la sovrapposizione di due rappresentazioni valide localmente. È, naturalmente, $u_{0} \leq u$. 
in $M_{n}$; mentre è sompre nullo l'integrale sopra un ciclo qualunque di una forma differenziale esatto $\left({ }^{3}\right)$.

Sia $\Gamma_{p}^{1}, \ldots, \Gamma_{p}^{R_{p}}\left(R_{p}\right.$ essendo il numero di BeTrI $p$-dimensionale di $\left.M_{n}\right)$ un sistema fondamentale per i $p$-cicli di $M_{n}$; e siano $k^{1}, \ldots, k^{R_{p}}$ numeri reali arbitrari. Il teorema di CARTAN-DE RHAM assicura precisamente l'esistenza di qualche forma $\omega_{p}$, regolare e integrabile in $M_{n}$, siffatta che risulti :

$$
\int_{\Gamma_{p}^{i}} \omega_{p}=k^{i} \quad\left(i=1, \ldots, R_{p}\right) .
$$

Introduzione di una metrica.

3. $\mathrm{E}$ noto che può costruirsi un modello della varietà $M_{n}$ immerso in uno spazio euclideo di dimensione conveniente, anch'esso di classe $u\left({ }^{7}\right)$. La metrica euclidea dell' ambiente subordina allora sul modello, e quindi sulla $M_{n}$, una metrica riemanniana di classe $u-1$.

D'altronde si può pervenire alla determinazione di una metrica riemanniana di classe $u-1$ su $M_{n}$ anche direttamente col metodo seguente, che potrebbe chiamarsi di sovrapposizione sfumata.

Siano $\mathfrak{J}_{n}^{1}, \ldots, \mathfrak{J}_{n}^{N}$ intorni atti a ricoprire $M_{n}$ (n. 2), e $\mathfrak{J}_{n}^{k}(k=1, \ldots, N)$ rappresentato sopra la regione ipersferica dello spazio euclideo $E_{n}^{k}\left(x_{1}^{k}, \ldots, x_{n}^{k}\right)$ :

$$
\sum_{i=1}^{n}\left(x_{i}^{k}\right)^{2}<\left(r^{k}\right)^{2}
$$

Si consideri su $\mathfrak{J}_{n}^{k}$ la metrica (trasformata conforme di quella di $E_{n}^{k}$ ) definita dalla forma quadratica

$$
\varepsilon^{k}\left(x_{1}^{k}, \ldots, x_{n}^{k}\right)\left[\left(d x_{1}^{k}\right)^{2}+\ldots+\left(d x_{n}^{k}\right)^{2}\right]
$$

dove con $\varepsilon^{k}$ s'indica una qualunque funzione positiva e di classe $u-1$ definita nella regione ipersferica, annullantesi insieme alle sue derivate fino all'ordine $u-1$ sul contorno; p. es. può assumersi $\varepsilon^{k}=\left[\left(r^{k}\right)^{2}-\Sigma_{i}\left(x_{i}^{k_{i}}\right)^{2}\right]^{u}$.

Si ottiene allora sopra l'intera $M_{n}$ una metrica che soddisfa ai requisiti richiesti, considerando, per ogni punto $P$ di $M_{n}$ ove si sovrappongano più intorni $\mathfrak{g}_{n}^{k}$, la metrica

$$
\sum_{k} \varepsilon^{k}\left[\left(d x_{1}^{k}\right)^{2}+\ldots+\left(d x_{n}^{k}\right)^{2}\right]
$$

l'indice $k$ percorrendo $\mathrm{i}$ valori corrispondenti agli intorni sovrapponentisi, e i differenziali $d x_{1}^{k}, \ldots, d x_{n}^{k}$ essendo espressi mediante quelli delle coordinate che si vogliono assumere in $P$. Si riconosce invero subito che la forma

(3) Sono queste ben note e immediate conseguenze della formula generale di GraENSToKes ; efr. p. es. F. Severx [6], pag. 384 .

(i) Ofr. H. Whitwey [8] e W. V. Hodge [2], pagg. 9 e 13. 
quadratica (2) risulta di classe $u-1$ in $M_{n}$, tenendo conto che le trasformazioni tra $\mathrm{i}$ sistemi di coordinate che possono assumersi in $P$ sono di classe $u$ (n. 2), quindi le trasformazioni tra $i$ differenziali di classe $u-1$, e che, quando $P$ muovendosi su $M_{n}$ esca da uno degli intorni $\mathfrak{g}_{n}^{k}$, la perdita del termine corrispondente nella somma (2) non altera la continuitâ dei coefficienti e delle loro derivate fino all'ordine $u-1$ della forma quadratica considerata, a causa delle proprieta delle funzioni $\varepsilon^{k}$. Inoltre la forma quadratica (2) è ovviamente definita positiva.

Nel seguito ei riferiremo alla metrien introdotia, od ad un'altra qualunque metrica di classe abbastanza alta, comunque nota in $M_{n}$.

\section{Preliminari topologici.}

4. Sia $\Delta_{n-p}^{1}, \ldots, \Delta_{n-p}^{R_{p}}$ una base per il gruppo di BETTI di dimensione $n-p$ in $M_{n}$, costituita da cicli che, come si è detto (n. 2), supponiamo di classe $u_{0}$ abbastanza alta. Consideriamo una qualunque base duale $\Gamma_{p}^{1}, \ldots, \Gamma_{p}^{R_{p}}$, in guisa che, per una fissata orientazione della varietà orientabile $M_{n}$, gl'indici di KRONECKER delle intersezioni valgano:

$$
\left(\Gamma_{p}^{i}, \Delta_{n-p}^{i}\right)=\delta^{i j}
$$

ove $\delta^{i j}=0$ per $i \neq j$, $\delta^{i l}=1$.

Fissiamo l'attenzione sopra un determinato ciclo $\Delta_{n-p}^{j}$ e sopra un suo punto $P$. Sia $G_{p}^{j}(P)$ la varieta geodetica $p$-dimensionale normale a $\Delta_{n-p}^{j}$ in $P$, lnogo cioè delle geodetiche dell' ambiente $M_{n}$ uscenti da $P$ secondo le $\infty^{p-1}$ direzioni ortogonali a $\Delta_{n-p}^{j}$. Sia inoltre $\sigma_{p-1}^{j}(P)$ la $(p-1)$-sfera geodetica tracciata su $G_{p}^{j}(P)$, di centro $P$ e raggio geodetico $r^{j}$ abbastanza piccolo, e $\Sigma_{p}^{j}(P)$ la $p$-cella geodetica, porzione di $G_{p}^{j}(P)$, delimitata da $\sigma_{p-1}^{j}(P)$ (contorno $\sigma_{p-1}^{j}$ incluso). Al variare del punto $P$ su $\Delta_{n-p}^{j}$ e tenendo costante $r^{j}$, $\Sigma_{p}^{j}(P)$ descrive intorno a $\Delta_{n-p}^{j}$ un tubo (o strato per $p=1$ ), $n$-dimensionale $T_{n}^{j}$, il cui contorno $l_{n-1}^{j}$ è descritto da $\sigma_{p-1}^{j}$.

Fissiamo sopra ogni $\Sigma_{p}^{j}(P)$ l'orientazione determinata da un $p$-edro $e_{p}$ di direzioni ortogonali a $\Delta_{n-p}^{j}$ in $P$, siffatto che, associato ordinatamente con un $(n-p)$-edro $e_{n-p}$ tangente in $P$ a $\Delta_{n-p}^{j}$ ed orientante secondo il suo verso $\Delta_{n-p}^{j}$, dia luogo ad un $n$-edro $e_{n}=\left(e_{p}, e_{n-p}\right)$ che orienta in $P$ la va. rietà $M_{n}$ concordemente all' orientazione già fissata per $M_{n}$. Risulta allora:

$$
\left(\Sigma_{p}^{j}, \Delta_{n-p}^{j}\right)=1
$$

Si osservi che, se $P^{\prime}$, $P^{\prime \prime}$ sono due punti di una stessa componente irriducibile del ciclo $\Delta_{n-p}^{j}$, le orientazioni cosi fissate su $\Sigma_{p}^{j}\left(P^{\prime}\right)$ e $\Sigma_{p}^{j}\left(P^{\prime \prime}\right)$ si mutano l'una nell'altra per continuità, quando si trasporti il punto $P$ da $P^{\prime}$ a $P^{\prime \prime}$ su tale componente e si segua la variazione continua di $\Sigma_{p}^{j}(P)$ : è una immediata conseguenza del fatto che la varietà $\Delta_{n-p}^{j}$ ed $M_{n}$ sono orientabili. 
14. Martinesis : Sulla costruzione osplicita rli forme differenziali, ecc. 101

Supponiamo ora, in un primo tempo, $\Delta_{n-p}^{j}$ irriducibile. Indichiamo con $\stackrel{*}{\Gamma}_{p}^{i}$ l'intersezione di $\Gamma_{p}^{i}$ con il tubo $T_{n}^{j}$. Lue varietà $\stackrel{*}{\Gamma}_{p}^{i}$ e le $\Sigma_{p}^{j}$ possono tutte con. siderarsi come cicli mod. $t_{n-1}^{j}$. Dimostriamo che risulta:

$$
\stackrel{*}{\Gamma}_{p}^{i} \approx \delta^{i j} \Sigma_{p}^{j} \text { in } T_{n}^{j}\left(\bmod . t_{n-1}^{j}\right) \text {. }
$$

Infatti, poichè la varietà $T_{n}^{j}-l_{n-1}^{j}$ può contrarsi su $\Delta_{n-p}^{j}$ (facendo tendere a zero il raggio del tubo), una base per il gruppo di BEITII $(n-p)$-dimensionale in $T_{p}^{j}-t_{n-1}^{j}$ eे costituita dal solo ciolo $\Delta_{n-p}^{j}$, e per stabilire la (5) basta verificare che

$$
\left(\Gamma_{p}^{i}-\delta^{i j} \Sigma_{p}^{j}, \Delta_{n-p}^{j}\right)=0\left({ }^{5}\right)
$$

ciò che è ben vero in base alle (3) e (4).

Nel caso in cui $\Delta_{n-p}^{j}$ si decomponga in più componenti irriducibili, $\Delta_{n-p}^{j}=\Delta_{n-p}^{j 1}+\ldots+\Delta_{n-p}^{j m}$ (delle quali eventualmente alcune sovrapposte), del pari accade che risulta $T_{n}^{j}=T_{n}^{j \mathrm{l}}+\ldots+T_{n}^{j m}$. Si ha inoltre

$$
\left(\Gamma_{p}^{i}, \Delta_{n-p}^{j k}\right)=\nu_{k}, \quad \nu_{1}+\ldots+v_{m}=\delta^{i j}
$$

e, ragionando come sopra in relazione a ciascun $T_{n}^{j k s}$, viene

$$
\stackrel{*}{\Gamma}_{p}^{i k k} \approx v_{k} \Sigma_{p}^{j}\left(P_{k}\right) \text { in } T_{n}^{j k}\left(\bmod . t_{n-1}^{j k}\right)
$$

dove con $\stackrel{*}{\Gamma}_{p}^{i l k}$ s'indica la parte di $\stackrel{*}{\Gamma}_{p}^{i}$ che appartiene a $T_{n}^{j k}$, e con $P_{k}$ un punto $q u a l u n q u e ~ d i \Delta_{n-p}^{j k}$. Sommando le $(7)$ risulta

$$
\stackrel{*}{\Gamma}_{p}^{i} \approx \nu_{1} \Xi_{p}^{j}\left(P_{4}\right)+\ldots+\nu_{m} \Sigma_{p}^{j}\left(P_{m}\right) \text { in } T_{n}^{j}\left(\bmod \cdot l_{n-1}^{j}\right) \text {. }
$$

5. Ciò posto, supponiamo d'aver costruito, per ogni ciclo $\Delta_{n-p}^{j}$ una forma differenziale di grado $p$, $\omega_{p}^{j}$, che diremo forma elementare associata. $a \Delta_{n-p}^{j}$, la quale soddisfaccia alle condizioni seguenti:

a) $\omega_{p}^{j}$ sia regolare nel tubo $T_{n}^{j}$, o, più precisamente, abbia coefficienti univocamente definiti e di classe $v \geq 1$;

b) i coefficienti di $\omega_{p}^{j}$ si annallino insieme alle loro derivate fino all'ordine $v$ sul contorno $t_{n-1}^{j}$ del tubo $T_{n}^{j}$;

c) $\omega_{p}^{j}$ sia integrabile $\left(d \omega_{p}^{j}=0\right)$;

d) $\operatorname{sia} \int \omega_{p}^{i}=1$.

$$
\Sigma_{p}^{i}(P)
$$

Una volta note $R_{p}$ forme elementari $\omega_{p}^{j}\left(j=1, \ldots, R_{p}\right)$, si ottengono subito forme che risolvono il problema esistenziale di CaRTan-De RHaM.

(5) Si ricordi che i cicli che soddisfanno ai teoremi di dualità rispetto ai $p$-cicli di $T_{n}^{j}$ mod. $t_{n-1}$, sono gli $(n-p)$-cicli ordinari di $T j_{n}-t_{n-1}$. Cfr. S. Lerschetz [3], pag. 178. 
S'immagini prolungata ogni $\omega_{p}^{j}$ in tatta $M_{n}$ come forma identicamente nulla fuori di $T_{n}^{j}$. La $\omega_{p}^{j}$ risulta allora, in base a $b$ ), di elasse $v$ e integrabile in $M_{n}$; e così pure accade per la forma

$$
\omega_{p}=\sum_{j=1}^{R_{p}} k^{j} \omega_{p}^{j},
$$

con $k^{j}$ costanti arbitrarie.

La (9) risolve il problema esistenziale. Infatti si ha:

$$
\int_{\Gamma_{p}^{i}}^{i} \omega_{p}=\sum_{j=1}^{R_{p}} k^{j} \int_{\Gamma_{p}^{i}} \omega_{p}^{j}=\sum_{j=1}^{R_{p}} k^{j} \int_{\substack{*_{i}^{i} \\ \Gamma_{p}^{i}}} \omega_{p}^{j} ;
$$

e poichè $\omega_{p}^{j}$ si annulla su $t_{n-1}^{j}$, per valutare gli ultimi integrali indicati, tenuto conto di $c$ ), possono sostituirsi alle varietà $i_{p}^{*}$ varietà omologhe in $T_{n}^{j}$ mod. $t_{n-1}^{j}$. Ricordando la $(5)$, o la più generale (8) insieme alla seconda delle (6), si ha quindi

$$
\int_{\substack{* \\ \Gamma_{p}^{i}}} \omega_{p}^{j}=\delta^{i j} \int_{\Sigma_{p}^{j}} \omega_{p}^{j}=\delta^{i j}
$$

e infine:

$$
\int_{\Gamma_{p}^{i}} \omega_{p}=\sum_{j=1}^{R_{p}} k^{j} \delta^{i j}=k^{i}
$$

come vuole la (1) del n. 2.

La costruzione di forme risolventi il problema esistenziale di CARTANDE RHAM, si riduce dunque essenzialmente alla costruzione di forme soddisfacenti le condizioni $a$ ), b), c), d). A quest'ultimo compito sono delicati i nn. seguenti.

\section{Caso semplice.}

6. Poichè tutto ciò che dovremo dire d'ora in avanti è lo stesso per $\mathbf{i}$ possibili valori dell' indice $j\left(=1, \ldots, R_{p}\right)$, ometteremo di apporlo ai simboli già usati $\Delta_{n-p}^{j}, \Sigma_{p}^{j}, T_{n}^{j}$, ecc.

Sopra la $p$-cella geodetica $\Sigma_{p}$ introduciamo un sistema di coordinate normali di RIEMANN $y^{1}, \ldots, y^{p}$, determinato da un $p$-edro $e_{p}(P)$ di direzioni ortogonali fra loro e a $\Delta_{n-p}$ in $P$, il quale sia concorde con l'orientazione fissata nel modo detto su $\Sigma_{p}(P)$ (n. 4). Indicheremo con $s=\left[\left(y^{\prime}\right)^{2}+\ldots+\left(y^{p}\right)^{2}\right]^{\frac{1}{2}}$ la distanza geodetica di un punto $Q\left(y^{1}, \ldots, y^{p}\right)$ di $\Sigma_{p}(P)$ dal punto $P$. 
Consideriamo poi, sopra la generica $\Sigma_{p}(P)$ di raggio $r$, la forma differenziale

$$
\omega_{p}=f\left(s^{2}\right) d\left(y^{1}, \ldots, y^{p}\right),
$$

dove $f\left(s^{2}\right)$ è una funzione di classe $v$ conveniente, definita nell'intervallo $\left(0, r^{2}\right)$, la quale soddisfaccia alle condizioni seguenti: 1) $f\left(s^{2}\right)$ si annulli nell'estremo $r^{2}$ insieme alle sue derivate fino all'ordine $v$;2) risulti :

$$
\int_{\Sigma_{p}} \omega_{p}=1\left({ }^{\hat{j}}\right) \text {. }
$$

Si noti che la forma $\omega_{p}$ è invariantivamente definita sopra ogni $\Sigma_{p}$, cioè non muta cambiando il $p$-edro $e_{p}$ che serve a determinare le coordinate normali $y^{k}, \ldots, y^{p}$. Se si immagina di rappresentare $\Sigma_{p}$ su uno spazio euclideo $E_{p}\left(y^{1}, \ldots, y^{p}\right)$, le trasformazioni di coordinate normali $y^{1}, \ldots, y^{p}$ possono invero rappresentarsi come rotazioni in $E_{p}$, le quali perciò non alterano nè il $p$-differenziale $d\left(x^{1}, \ldots, y^{p}\right)$ nè $s$, attesi i loro significati geometrici di elemento di volume e di distanza in $E_{p}$. D'altronde l'invarianza della (10) è formalmente immediata.

Orbene, tenderemo alla costruzione di una forma elementare associata $a \Delta_{n-p}$ (n. 5), la quale sia definita nel tubo $T_{n}$ descritto dalle $\Sigma_{p}(P)$ al variare di $P$ su. $\Delta_{n-p}$, e si riduca sopra ogni $\Sigma_{p}(P)$ alla forma $\omega_{p}$.

Supponiamo per un momento che, mentre $P$ descrive tutto il ciclo $\Delta_{n-p}$, si possa assegnare una legge che permetta di trasportare in modo ovunque continuo e regolare il $p$-edro ortogonale $e_{p}$ di direzioni normali a $\Delta_{n-p}$; diciamo allora che si verifica il caso semplice. Discuteremo in seguito (nn. 16, 17) le condizioni per il suo verificarsi, limitandoci per ora ad osservare che ciò accade ovviamente per $p=1$ e per $p=n-1$.

Nel caso semplice un punto qualunque $Q$ del tubo $T_{n}$ può individuarsi mediante le $p$ coordinate $y^{1}, \ldots, y^{p}$ sulla $\Sigma_{p}(P)$ passante per $Q$, definite a partire dallo $e_{p}(P)$ che determinato secondo la legge predetta, e mediante altre $n-p$ coordinate $z^{p+1}, \ldots, z^{n}$ atte ad individuare la posizione di $P$

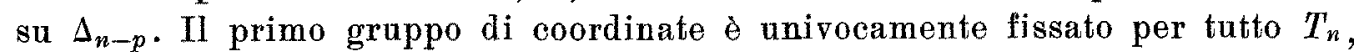
mentre il secondo gruppo ha validità soltanto locale, e deve perciò assumersi differentemente da luogo a luogo.

Si ottengono allora immediatamente forme elementari. La $\omega_{p}$ stessa, così come è scritta in (10), ১̀ tale. Infatti la (10) può pensarsi come una forma differenziale nelle coordinate $\left(y^{a}, \approx^{x}\right)(a=1, \ldots, p ; \alpha=p+1 \ldots, n)$, nella quale non appaiono le $z^{x}$; onde essa risulta definita in tutto il tubo $T_{n}$. La

(6) Si costruiscono ovviamente funzioni che verificano le condizioni 1), 2). Basta p. es. assumere $f\left(s^{2}\right)=\frac{1}{\lambda(r)} f_{0}\left(s^{2}\right)$, con $f_{0}\left(s^{2}\right)=\left(r^{2}-s^{2}\right)^{y+1}$ e $\lambda(r)=\int_{\Sigma_{p}} f_{0}\left(s^{2}\right) d\left(y^{1}, \ldots, y^{p}\right)>0$. 
integrabilità della forma ̀̀ d'altronde orvia, perchè il suo differenziale esterno è

$$
\sum_{a=1}^{p} \frac{\partial f\left(s^{2}\right)}{\partial y^{a}} d\left(y^{a}, y^{1}, \ldots, y^{p}\right)+\sum_{\alpha=p+1}^{n} \frac{\partial f\left(s^{2}\right)}{\partial z^{x}} d\left(z^{x}, y^{1}, \ldots, y^{p}\right)
$$

del quale ciascun termine è nullo, perchè nella prima somma sono nulli tutti i $(p+1)$-differenziali, $\theta$ nella seconda tutti i coefficienti. Se si tien conto infine delle proprietà della funzione $f\left(s^{2}\right)$ e della (11), si conclude che la $\omega_{p}$ soddisfa proprio a tutte le condizioni che definiscono una forma elementare di classe $v$ (n. 5).

Osservazrone 1a $1^{\mathrm{a}}$ - Affinchè i coefficienti della forma elementare co. struita risultino in effetti di classe $v$ (come la $f\left(s^{2}\right)$ scelta) rispetto alle coordinate generali $x_{1}, \ldots, x_{n}$ in $M_{n}$, è ovvio che bisogna supporre convenientemente alte le classi $u, u_{0}, t$ rispettivamente di $M_{n}, \Delta_{n-p}$ e della legge di trasporto del $p$-edro $e_{p}$ che si suppone nota. Non v'è difficoltà per determinare limitazioni inferiori per $u, u_{0}, t$ sufficienti affinchè la classe della forma sia pro. prio $v$. Osservazioni analoghe sottintendiamo nel seguito trattando il caso generale, in cui, avvertiamo, basterà supporre limitazioni inferiori per $u$ e $u_{0}$.

OSSERVAZIONe $2^{\mathrm{a}}$. - Benchè la forma $\omega_{p}$ sia invanriantivamente definita sopra ogni $\Sigma_{p}$, la forma elementare cui si è giunti in $T_{n}$, che ha pure la medesima espressione di $\omega_{p}$, non è ovviamente invariante (per $p>1$ ), in quanto dipende dalla supposta legge di trasporto del $p$-edro $e_{p}$. Le forme elementari che costruiremo trattando il caso generale, sono invece sempre invarianti, come si è detto al n. 1. D'altronde, nel caso semplice, il fatto di partire da una forma definita in modo invariante sopra ogni $\Sigma_{p}$ non ha alcuna importanza ai fini del ragionamento sviluppato. Il ragionamento stesso può invero ripetersi immutato se si sostituisce $f\left(s^{2}\right)$ con una qualunque funzione $f\left(y^{4}, \ldots, y^{p}\right)$, che si annulli sul contorno di $\Sigma_{p}$ insieme alle sue derivate fino all'ordine $v$ e soddisfaccia ancora la (11).

OSSERVAZione $3^{a}$. - Allorchè sia riducibile il ciclo $\Delta_{n-p}=\Delta_{n-p}^{1}+\ldots+\Delta_{n-p}^{m}$, col procedimento indicato si viene a costruire una forma elementare entro ciascun tubo $T_{n}^{k}$ componente di $T_{n}=T_{n}^{1}+\ldots+T_{n}^{m}$ (n. 4); la quale forma deve poi estendersi come identicamente nulla in tutta la $M_{n}$ (n. 5). La forma che deve assumersi come forma elementare associata all'intero ciclo $\Delta_{n-p}$, non è altro, in questo caso, che la somma delle forme relative ai cicli $\Delta_{n-p}^{k}$. La stessa osservazione è valida per il seguito.

Caso generale. Preliminare constatuzione negativa.

7. Passiamo alla considerazione del caso generale. Pur prescindendo dalla validità del caso semplice, si può sempre assegnare una legge regolare di trasporto del $p$-edro ortogonale $e_{p}(P)$, limitatamente però al variare del punto $P$ in un intorno $\mathfrak{J}_{\Delta}\left(P_{0}\right)$ di un fissato $P_{0}$ su $\Delta_{n-p}$. Non appena sia $p>1$ vi sono infinite possibilità di far ciò. 
Il complesso delle coordinate $\left(y^{a}, z^{x}\right)$ permette allora di rappresentare ogni punto $Q$ appartenente alla porzione del tubo $T_{n}$ che è descritta da $\Sigma_{p}(P)$ al variare di $P$ in $\mathfrak{J}_{\Delta}\left(P_{0}\right)$. Se si cambiano la legge di trasporto par lo $e_{p}(P)$ e i parametri $z^{p+1}, \ldots, z^{n}$, si opera una trasformazione di coordinate del tipo:

$$
\left\{\begin{array}{l}
y^{a}=c_{\alpha^{\prime}}^{\alpha}\left(\bar{z}^{p+1}, \ldots, \bar{z}^{n}\right) \bar{y}^{a^{\prime}} \\
z^{z}=z^{\alpha}\left(\bar{z}^{p+1}, \ldots, \bar{z}^{n}\right)
\end{array} \quad\left(a, a^{\prime}=1, \ldots, p ; \alpha=p+1, \ldots, n\right),\right.
$$

ove il determinante delle $c_{. a^{\prime}}^{a}$, funzioni delle $\bar{z}^{p+1}, \ldots, \bar{z}^{n}$, ¿ ortogonale positivo, ed è sottinteso il segno di somma rispetto all'indice $a^{\prime}$.

Ciò posto, conviene richiamare l'attenzione su quanto segue. Se si ragiona come al n. 6, ma limitatamente alla regione parziale di $T_{n}$ ora considerata, la espressione (10) fornisce una forma differenziale che può pensarsi definita e integrabile in tale regione. Considerato allora un numero finito di intorni del tipo di $\mathfrak{g}_{\Delta}\left(P_{0}\right)$. ricoprenti $\Delta_{n-p}$, si ottiene in corrispondenza una suddivisione di $T_{n}$ in regioni parziali, in ciascuna delle quali risulta similmente definita una forma differenziale integrabile. Tenuto conto della invarianza della (10) per trasformazioni di coordinate normali $y^{1}, \ldots, y^{p}$ : si potrebbe allora essere indotti a credere di aver così ottenuto una unica forma differenziale regolare ed integrabile in tutto il tubo $T_{n}$.

Ma così non è in generale. Ciascuna forma definita in una regione parziale di $T_{n}$ dipende invero essenzialmente dalla legge usata nel corrispondente intorno $\mathfrak{J}_{\Delta}\left(P_{0}\right)$ di $\Delta_{n-p}$ per trasportare il $p$-edro $e_{p}$. In un punto $Q$ di $T_{n}$ che sia comune a due regioni parziali ivi sovrapponentisi, indicate con $y^{a}$ e $\bar{y}^{a}$ le coordinate normali relative alle due regioni, si hanno le due forme

$$
\omega_{p}=f\left(s^{2}\right) d\left(y^{1}, \ldots, y^{p}\right), \quad \bar{\omega}_{p}=f\left(s^{2}\right) d\left(\bar{y}^{1}, \ldots, \bar{y}^{p}\right) .
$$

Se ora si vuole esprimere p. es. la prima forma $\omega_{p}$ con le coordinate della seconda, bisogna eseguire sul $p$-differenziale $d\left(y^{1}, \ldots, y^{p}\right)$ una sostituzione ortogonale $\mathrm{i}$ cui coefficienti dipendono in generale dalle coordinate $z^{p+1}, \ldots, z^{n}$ del punto $P$ variabile su $\Delta_{n-p}$, onde la forma trasformata viene a contenere dei termini nei differenziali delle $z^{\alpha}$, e non coincide così con la $\bar{\omega}_{p}$.

\section{Il parallelismo $W$. Caso di integrabilità.}

8. Premessa questa constatazione negativa, osserviamo che, pur sempre prescindendo dalla validità del caso semplice, può in ogni caso determinarsi una legge di trasporto infinitesimo, ovunque regolare su $\Delta_{n-p}$, intrinsecamente (e quindi univocamente) definita, la quale permetta di trasportare un $p$-edro $e_{p}(P)$ di vertice $P$, ortogonale a $\Delta_{n-p}$, in un $p$-edro $e_{p}\left(P^{\prime}\right)$ congruente, ortogonale a $\Delta_{n-p}$ in un punto $P^{\prime}$ infinitamente vicino a $P$.

Invero, sia $v$ un vettore ortogonale a $\Delta_{n-y}$ in $P$, e $v^{\prime}$ il vettore trasportato in $P^{\prime}$ col parallelismo di Levi-CIvita relativo alla metrica dell'am. biente $M_{n}$. Se si considera l'operazione che muta $v$ nel vettore $v^{*}$, che è 
componente normale $a \Delta_{n-p}$ del vettore $v^{\prime}$, si ottiene un parallelismo che permette di trasportare vettori ortogonali a $\Delta_{n-p}$ in vettori ortogonali. Chiameremo questo parallelismo, che è stato per la prima volta considerato da H. WEYL [7], parallelismo W. Ebbene, trasportando il $p$-edro $e_{p}$ mediante il parallelismo $W$, si ha appunto una legge di trasporto infinitesimo del tipo desiderato, giacchè si verifica facilmente che il parallelismo $W$ conserva gli angoli tra vettori (ciò she è del resto geometricamente evidente, tenuto conto che della stessa proprietà gode il parallelismo di Levi-Crvima).

Supponiamo, in un primo tempo, che il parallelismo $W$ sia integrabile, vale a dire che, con una circolazione del vertice $P$ su $\Delta_{n-p}$ (limitata, se occorre, ad un intorno abbastanza piccolo su $\Delta_{n-p}$ ), il $p$-edro $e_{p}$ trasportato per parallelismo ritorni in se stesso. Ciò accade certamente quando sia $p=1$ ovvero $p=n-1$, giacchè, nel primo caso lo $e_{p}$ si riduce all' unica normale a $\Delta_{n-p}$ nel punto $P$ variabile, e nel secondo easo, riducendosi $\Delta_{1}$ ad una linea, le circolazioni locali di $P$ su $\Delta_{1}$ si riducono a percorsi di andata e ritorno sullo stesso tratto di linea. In quest'ultimo caso tuttavia, la circola. zione di $P$ sull'intera linea chiusa $\Delta_{1}$ non è escluso possa produrre una rotazione dello $(n-1)$-edro $e_{n-1}$. Comunque, sia per i valori indicati di $p$, sia per ogni altro valore di $p$ quando il parallelismo $W$ risulti integrabile, la situazione è quasi la medesima di quella che si presenta nel caso semplice (n. 6).

Infatti, nell'attuale ipotesi, mercè il parallelismo $W$ si può, partendo da un determinato $e_{p}\left(P_{0}\right)$ di vertice $P_{0}$ comunque fissato su $\Delta_{n-p}$, trasportare il $p$-edro in modo regolare e univocamente determinato, se non addirittura su tutta la varietà $\Delta_{n-p}$, almeno a tutto in intorno finito $\mathfrak{I}_{\Delta}\left(P_{0}\right)$ del punto $P_{0}$, $\mathrm{E}$ tenuto conto del fatto che il paralleliamo $W$ è determinato intrinsecamente, si può in questo caso sviluppare con successo il ragionamento indicato al n. 7 , giacchè le due forme $\omega_{p}, \bar{\omega}_{p}$ ivi considerate vengono di fatto a coincidere, in quanto il passaggio dall'una all'altra si fa attualmente mediante una sostituzione ortogonale a coefficienti costanti.

\section{Forme inviluppo.}

9. In generale il parallelismo $W$ non è però integrabile, come non lo è il parallelismo di LEVI-CIVITA. Ecco allora come può ugualmente ginngersi alla costruzione di una forma differenziale definita e regolare in $T_{n}$, che si riduca alla (10) sopra ogni $\Sigma_{p}$.

Fissato un punto $P_{0}$ di $\Delta_{n-p}$ consideriamo le $\infty^{n-p-1}$ geodetiche entro $\Delta_{n-p}$ uscenti da $P_{0}$, ovvero un qualunque altro sistema di linee tracciate su $\Delta_{n-p}$ e uscenti da $P_{0}$ secondo tutte le possibili direzioni : indicheremo un $t_{a}$ sistema di linee con $\{l\}_{P_{0}}$. Assegnato un $p$-edro $e_{p}\left(P_{0}\right)$ di vertice $P_{0}$, questo può trasportarsi col parallelismo, usando come linee di trasporto quelle del sistema $\{l\}_{P_{0}}$, a tutto un intorno $\mathfrak{J}_{\Delta}\left(P_{\mathrm{v}}\right)$ in modo univocamente determinato. 
Ripetendo il ragionamento del n. 7, la forma (10) dà luogo allora ad una forma integrabile definita in una regione parziale del tubo $T_{n}$, che ha la stessa espressione formale della $(10)$ e che indicheremo con $\omega_{p}^{P_{0}}$. Ciò può ripetersi a partire da ogni punto $P_{0}$ scelto su $\Delta_{n-p}$, ottenendosi così altrettante forme differenziali $\omega_{p}^{P_{0}}$. $\dot{\mathrm{E}}$ ormai ben chiaro peró che, se il parallelismo $W$ non è integrabile, le forme costruite non coincidono affatto, anche se si assume come sistema di linee $\{l\}_{P_{0}}$ per ogni $P_{0}$ su $\Delta_{n-p}$ il sistema intrinsecamente determinato delle geodetiche per $P_{0}$. Infatti, in ogni caso, ciascuna delle forme $\omega_{p}^{P_{0}}$ costruite viene a dipendere dal punto $P_{0}$ scelto come centro della costruzione predetta. Quel che soltanto può asserirsi, in virtù dell'invarianza per rotazioni della (10), è che ciasouna forma costruita non dipende dal particolare $e_{p}\left(P_{0}\right)$ fissato nel centro $P_{0}$.

Ebbene, perverremo lo stesso ad una forma univocamente definita in tutto il tubo $T_{n}$, considerando quella che diremo la forma inviluppo $\tilde{\omega}_{p}$ delle forme $\omega_{p}^{P_{0}}$. Se $Q_{0}$ è un punto comunque variabile nel tabo $T_{n}$ e $\Sigma_{p}\left(P_{0}\right)$ la $p$-cella geodetica per esso, definiamo come $\tilde{\omega}_{p}$ la forma che si riduce in ogni punto $Q_{0}$ alla forma $\omega_{p}^{P_{0}}$.

Si capisce che la forma inviluppo $\tilde{\omega}_{p}$ dipende soltanto dalla, $\omega_{p}$ e dal parallelismo $W$ e non dai sistemi di linee scelti $\{l\}_{P_{0}}$ per ogni $P_{0}$ sù $\Delta_{n-p}$. Infatti, basta per convincersene tener presente che $\mathrm{i}$ coefficienti di una forma differenziale si comportano in una trasformazione di variabili come tensori; per modo che, in an dato punto, la relazione che intercede tra $i$ valori ivi assunti dai coefficienti di due forme l'una trasformata dell'altra dipende soltanto dai valori delle derivate prime nel punto stesso delle funzioni che rappresentano la trasformazione. Del resto può ottenersi l'espressione esplicita della $\tilde{\omega}_{p}$ nel modo seguente.

Per evitare confusione, indichiamo con $t^{1}, \ldots, t^{p}$ le coordinate normali in $\Sigma_{p}(P)$ determinate dal $p$-edro $e_{p}(P)$, che si suppone ottenuto da un fissato $\bar{e}_{p}\left(P_{0}\right)$ mediante trasporto col parallelismo $W$ eseguito lungo le linee $\{l\}_{P_{0}}$, essendo $P$ nell' intorno $\mathfrak{g}_{\Delta}\left(P_{0}\right)$. Assunte allora $\left(t^{a}, z^{\alpha}\right)$ come coordinate atte a descrivere la porzione di $T_{n}$ generata da $\Sigma_{p}(P)$, la forma $\omega_{p}^{P_{0}}$ è data sempli. cemente da: con $s^{2}=\left(t^{1}\right)^{2}+\ldots+\left(t^{p}\right)^{2}$.

$$
\omega_{p}^{P_{0}}=f\left(s^{2}\right) d\left(t^{1}, \ldots, t^{p}\right)
$$

Indichiamo invece con $\left(y^{a}, z^{x}\right)$ il riferimento, valido nella stessa porzione di $T_{n}$, già determinato al n. 7 mediante il trasporto con legge generica del $p$-edro $e_{p}(P)$ col quale si definiscono le coordinate $y^{1}, \ldots, y^{p}$ su $\Sigma_{p}(P)$. A causa del carattere invariante rilevato per la $\omega_{p}$ (n. 6), possiamo supporre, senza che occorra percio di alterare la $(13)$, che per $P=P_{0}$ lo $\bar{e}_{p}\left(P_{0}\right)$ che serve a definire le coordinate $t^{1}, \ldots, t^{p}$ sopra la $\Sigma_{p}\left(P_{0}\right)$ coincida con lo $e_{p}\left(P_{0}\right)$ che determina le coordinate $y^{1}, \ldots, y^{p}$ sopra la $\Sigma_{p}\left(P_{0}\right)$ stessa. Sopra tale $\Sigma_{p}\left(P_{0}\right)$ (e, in generale, soltanto su questa) è allora $y^{a}=t^{a}$. 
Vogliamo esprimere la forma inviluppo $\tilde{\omega}_{p}$ nel riferimento $\left(y^{a}, z^{x}\right)$. All'uopo associamo ad un punto $Q_{0}\left(y^{a}, z^{x}\right)$ di $\Sigma_{p}\left(P_{0}\right)$, il vettore contravariante applicato in $P_{0}$ e ortogonale a $\Delta_{n-p}, v$, di componenti $y^{\prime}, \ldots, y^{p}, 0, \ldots, 0$ nel riferimento $\left(y^{a}, z_{i}^{x}\right)$. Si tratta di un vettore tangente in $P_{0}$ alla geodetica $P_{0} Q_{0}$. Siano $\delta y^{1}, \ldots, \delta y^{p}, 0, \ldots, 0$ le componenti contravarianti del differenziale cova. riante di $v$ rispetto al parallelismo $W$. Si ha ovviamente, ricordata la definizione di $W($ n. 8),

$$
\delta y^{a}=d y^{a}+\left\{\begin{array}{c}
a \\
b \beta
\end{array}\right\}_{0}^{b} d z^{\beta}
$$

dove $\left\{\begin{array}{c}a \\ b \beta\end{array}\right\}_{0}$ sono simboli di CHRIsTOFFeL di $2^{a}$ specie relativi alla metrica dell'ambiente $M_{n}$ espressa in coordinate $\left(y^{a}, z^{x}\right)$; coll' indice 0 intendiamo che $\mathrm{i}$ simboli stessi siano valutati nel punto $\left(0, z^{x}\right)$. Nella (14) sono sottintesi i segni di somma rispetto agl' indici $b=1, \ldots, p$ e $\beta=p+1, \ldots, n$.

Ciò pósto, facciamo vedere che la forma $\tilde{\omega}_{p}$ si ottiene dalla (10) sostituendo il $p$-differenziale $d\left(y^{1}, \ldots, y^{p}\right)$, espresso come prodotto esterno di differenziali semplici

$$
d\left(y^{1}, \ldots, y^{p}\right)=d y^{1} \times \ldots \times d y^{p}\left({ }^{7}\right),
$$

con il prodotto esterno dei differenziali covarianti considerati. Precisamente si ha

$$
\tilde{\omega}_{p}=f\left(s^{2}\right) \delta y^{1} \times \ldots \times \delta y^{p} .
$$

Infatti, tenuto conto dell' invarianza per trasformazioni di variabili delle operazioni di prodotto esterno e di differenziazione covariante, la (15) nelle coordinate $\left(t^{a}, z^{x}\right)$ diviene

$$
\tilde{\omega}_{p}=f\left(s^{2}\right) \delta t^{1} \times \ldots \times \delta t^{p} .
$$

D'altronde, per i punti $Q_{0}$ di $\Sigma_{p}\left(P_{0}\right)$ è evidentemente $\delta t^{a}=d t^{a}$, quindi in ogni tal punto $Q_{0}$ il secondo membro della (16) coincide col secondo membro della (13). In formule:

$$
\tilde{\omega}_{p}\left(Q_{0}\right)=\omega_{p}^{P_{0}}\left(Q_{0}\right) ;
$$

che è quanto si voleva provare.

La forma inviluppo $\dot{\omega}_{p}$, della quale la (15) fornisce l'espressione espli. cita, è, di sua natura, definita invariantivamente, e quindi univocamente, in lutto il tubo $T_{n}$, e soddisfa alla condizione di ridursi alla (10) sopra ogni $\Sigma_{p}$. Tuttavia la $\tilde{\omega}_{p}$ non è ancora una forma che possa in ogni caso assumersi come forma elementare associata al ciclo $\Delta_{n-p}$, in quanto non v'è in generale alcuna ragione perchè là $\tilde{\omega}_{p}$ risulti integrabile. (Essa risulta certamente integrabile quando è integrabile il parallelismo $W$, perchè allora tutte le forme $\omega_{p}^{P_{0}}$ coincidono fra loro e con la $\tilde{\omega}_{p}$ ).

(7) Indichiamo col segno $\times$ l'operazione di prodotto esterno. 
Forme fondamentali $\tilde{\mathbf{\Omega}}_{p}, \tilde{\Theta}_{p-1}$.

10. Per giungere ad una forma sempre integrabile possiamo procedere cosi.

Allo scopo di poter usare nel seguito di una serittura più spedita, consideriamo accanto alla (10) anche la semplice forma

$$
\mathbf{Q}_{p}=d\left(y^{1}, \ldots \cdot y^{p}\right)
$$

invariantivamente definita su una generica $\Sigma_{p}(P)$, e la forma inviluppo da essa determinata

$$
\tilde{\Omega}_{p}=\delta y^{i} \times \ldots \times \delta y^{p},
$$

che è invece invariantivamente definita nel tubo $T_{n}$. 亡 allora:

$$
\omega_{p}=f\left(s^{*}\right) \mathbf{\Omega}_{p}, \quad \tilde{\omega}_{p}=f\left(s^{z}\right) \tilde{\Omega}_{p} .
$$

Sopra $\Sigma_{p}\left(P\right.$, la forma $\Omega_{p}$ è differenziale esatto della forma di grado $p-1$ :

$\Theta_{p-1}=\frac{1}{p}\left\{y^{1} d\left(y^{2}, \ldots, y^{p}\right)-y^{2} d\left(y^{1}, y^{3}, \ldots, y^{p}\right)+\ldots+(-1)^{p-1} y^{p} d\left(y^{1}, \ldots, y^{p-1}\right)\right\}$.

In formule, si ha cioè :

$$
d \Theta_{p-1}=\Omega_{p} .
$$

Come la $\Omega_{p}$, cosî anche la $\Theta_{p-1}$ è una forma invariantivamente definita sopra $\Sigma_{p}(P)$ (n. 6). Lo si verifica subito eseguendo una sostituzione ortogonale sulle $y^{1}, \ldots, y^{p}$, oppure osservando che, se si rappresenta $\Sigma_{p}$ sopra uno spazio euclideo $E_{p}\left(y^{1}, \ldots, y^{p}\right)$ come al n. 6 , la $\Theta_{p-1}$ esprime il volume della piramide che ha vertice nell'origine delle coordinate e base costituita dal parallelepipedo individuato da $p-1$ vettori infinitesimi uscenti dal punto di coordinate $\left.\left(y^{1}, \ldots, y^{p}\right){ }^{8}\right)$.

Ora possiamo ripetere per la forma $\Theta_{p-1}$ quanto si è detto al n. 9 deducendone la forma inviluppo:

$$
\tilde{\Theta}_{p-1}=\frac{1}{p}\left\{y^{1} \delta y^{2} \times \ldots \times \delta y^{p}-\ldots . .+(-1)^{p-1} y^{p} \delta y^{1} \times \ldots \times \delta y^{p-1}\right\} .
$$

La forma $\tilde{\Theta}_{p-1}$ di grado $p-1$, e così pure la forma di grado $p$ che ne ¿̀ differenziale esterno $d \tilde{\Theta}_{p-1}$, risultano invariantivamente (e univocamente) definite nel tubo $T_{n}$. La forma $d \tilde{\Theta}_{p-1}$ risulta inoltre integrabile, in base al citato teorema di PoINCARÉ (n. 2). Osserviamo ancora ohe, se si considera la

(8) Infatti, immaginando i $(p-1)$-differenziali che appaiono nella $\theta_{p-1}$ determinati dai $p-1$ vettori considerati, il volume della piramide suddetta è la $p$-ma parte di quello del parallepipedo individuato dai precedenti $p-1$ vettori infinitesimi e dal vettore di componenti $-y^{1}, \ldots,-y^{p}$, applicato anch' esso nel punto $\left(y^{1}, \ldots, y^{p}\right)$. D'altronde il volume del parallepipedo uguaglia (a meno del segno) il determinante formato dalle componenti di tutti i $p$ vettori : ciò̀ quanto è in parentesi nel secondo membro della (20). Per $p=2$, nel piano cartesiano $(x, y)$ si ha la ben nota forma pfaffiana $\theta_{1}=1 / 2(x d y-y d x)$ il cui integrale sopra una linea chiusa esprime l'area rachiusa dalla curva. 
forma inviluppo $\tilde{\Theta}_{p-1}$ sopra una $\Sigma_{p}$, si ha $\left(\tilde{\Theta}_{p-1}\right)_{\Sigma_{p}}=\theta_{p-1}$; onde, tenuto conto che $\left(d \tilde{\Theta}_{p-1}\right) s_{p^{\prime}}=d\left(\tilde{\Theta}_{p-1}\right) \mathrm{s}_{p}$ e della (21), risulta :

$$
\left(d \tilde{\Theta}_{p-1}\right)_{\Sigma_{p}}=\Omega_{p} \text {. }
$$

Se $g\left(s^{2}\right)$ è una fissata funzione di $s^{2}$, le stesse proprietà ora indicate per $\Theta_{p-1}, \tilde{\Theta}_{p-1}, d \tilde{\Theta}_{p-1}$, spettano rispettivamente alle forme $: g\left(s^{2}\right) \Theta_{p-1}, g\left(s^{2}\right) \tilde{\Theta}_{p-1}$, $d\left[g\left(s^{2}\right) \tilde{\Theta}_{p-1}\right]$.

Differenziando esternamente la forma $g \Theta_{p-1}$, considerata sopra una generica $\Sigma_{p}$, si ha poi :

$$
d\left[g \theta_{p-4}\right]=g^{\prime}\left(s^{2}\right) d s^{2} \times \Theta_{p-1}+g\left(s^{2}\right) d \Theta_{p-\frac{1}{2}} ;
$$

percio, ricordando la (21) e osservando che

$$
\Omega_{p}=\frac{p}{2} \frac{1]}{s^{2}} d s^{2} \times \Theta_{p-1},
$$

risulta :

$$
d\left[g \Theta_{p-1}\right]=\left\{\frac{2}{p} s^{2} g^{\prime}\left(s^{*}\right)+g\left(s^{2}\right)\right\} \mathbf{\Omega}_{p}
$$

Assumiamo per $g\left(s^{2}\right)$ un integrale, per ora non precisato, della equazione differenziale:

$$
\frac{2}{p} s^{2} g^{\prime}\left(s^{2}\right)+g\left(s^{2}\right)=f\left(s^{2}\right)
$$

In base alla prima delle (19) si ha allora:

$$
d\left[g \Theta_{p-1}\right]=f \Theta_{p}=\omega_{p},
$$

donde si trae, analogamente a sopra,

$$
\left(d\left[g \tilde{\Theta}_{p-1}\right]\right)_{\Sigma_{p}}=f \boldsymbol{\Theta}_{p}=\omega_{p} .
$$

La forma $d\left[g \tilde{\Theta}_{p-1}\right]$ è dunque una forma di grado $p$, invariantivamente definita nel tubo $T_{n}$, ivi integrabile, che si riduce alla $\omega_{p}$ sopra ogni $\Sigma_{p}$. Tale forma può allora assumersi come forma elementare associata al ciclo $\Delta_{n-p}$ ?

\section{Esame critico.}

11. È necessario esaminare con un po' d'attenzione la domanda finale del n. precedente.

La forma $d\left[g \widetilde{\Theta}_{p-1}\right]$ dipende dalla scelta di un integrale, $g\left(s^{2}\right)$, dell' equazione differenziale (24). L' integrale generale della (24), per $s$ variabile nell' intervallo $(0, r)$ che a noi interessa, è

$$
c s^{-p}+\frac{p}{2} s^{-p} \int_{0}^{s^{z}} f\left(s^{q}\right) s^{p-2} d s^{2},
$$

con $c$ costante arbitraria. 
Si verifica facilmente che per $c=0$ la (27) fornisce un integrale regolare, anzi l'unico integrale regolare,

$$
g_{0}\left(s^{2}\right)=\frac{p}{2} s^{-p} \int_{0}^{s^{2}} f\left(s^{2}\right) s^{p-2} d s^{2}
$$

nonostante l'apparente indeterminazione per $s^{*}=0$. La funzione $g_{1}\left(s^{2}\right)$ risulta precisamente della stessa classe $v$ di $f\left(s^{2}\right)$ in tutto l'intervallo $\left(0, r^{2}\right)(n, 6)$. Con siffatta scelta della funzione $g\left(s^{2}\right)=g_{0}\left(s^{2}\right)$, la forma $g_{0} \tilde{\Theta}_{p-1}$ ̀े regolare nel tubo $T_{n}$ e così pure la $d\left[g_{0} \tilde{\Theta}_{p-1}\right]$. Tenuto conto della $(26)$ si è allora tentati di pensare di avere in questo modo ottenuto effettivamente una forma elementare associata al ciclo $\Delta_{n-p}$. Ma ciò non è vero, in generale. Infatti, $d\left[g_{9} \tilde{\Theta}_{p-1}\right]$ soddisfa sì alle condizioni $a$ ), c), d) del n. 5 , ma la condizione $b$ ), concernento l'annullamento dei coefficienti della forma sul contorno del tabo $T_{n}$, è soddisfatta dalla forma $\left(d\left[g_{0} \tilde{\Theta}_{p-1}\right]\right) \Sigma_{p}$ considerata sopra una $\Sigma_{p}$ (cioè dalla $\omega_{p}$ ), e non dalla $\left(d\left[g_{0} \tilde{\Theta}_{p-1}\right]\right)$ liberamente considerata nei punti del contorno di $T_{n}$. In altri termini, la (26) ci dice soltanto che si annullano sul contorno del tubo il coefficiente del $p$-differenziale $d\left(y^{1}, \ldots, y^{p}\right)$ che appare in $d\left[g_{0} \tilde{\Theta}_{p-1}\right]$, mentre nulla ci dice circa l'annullarsi dei coefficienti degli altri $p$-differenziali contenenti i differenziali $d z^{x}$. (9).

Anzichè considerare l' integrale regolare che si ottiene per $c=0$, possiamo assumere $c=-\frac{p}{2} \int_{0}^{r^{2}} f\left(s^{2}\right) s^{p-2} d s^{2}$, ottenendo l'integrale

$$
g_{1}\left(s^{2}\right)=\frac{p}{2} s^{-p} \int_{r^{2}}^{s^{2}} f\left(s^{2}\right) s^{p-2} d s^{2} \quad\left(s^{2} \leq r^{2}\right) .
$$

La funzione $g_{1}\left(s^{2}\right)$ è singolare in $s^{2}=0$, ma, ad eccezione di questo punto, risulta di classe $v+1$ nell' intervallo $\left(0, r^{2}\right)$, essendo ivi $f\left(s^{2}\right)$ di classe $v$. Inoltre, avuto riguardo alla condizione 1) supposta per $f\left(s^{2}\right)$ (n. 6), risulta che $g_{1}\left(s^{2}\right)$ si annulla per $s^{2}=r^{2}$ insieme alle sue derivate fino allordine $v+1$.

Ne segue subito che la forma $d\left[g_{1} \tilde{\Theta}_{p-1}\right]$ soddisfa effettivamente alla condizione b)-del n. 5 . Le condizioni $c) d$ ) sono anche soddisfatte dalla $d\left[g \tilde{\Theta}_{p-1}\right]$. Quanto alla condizione $a$ ) essa è certamente soddisfatta nei punti di $T_{n}-\Delta_{n-p}$, ma non si può asserire che sia soddisfatta nei punti di $\Delta_{n-p}$, poichè ivi i coefficienti di $g_{1} \tilde{\Theta}_{p-1}$ risultano singolari in dipendenza della singolarità

$\left({ }^{9}\right)$ Che di fatto non svaniscano in generale anche questi coefficienti, può verificarsi su esempi. Se $p=2$, p. es., la (41) del successivo n. 13, nella quale si ponga $g_{0}\left(s^{2}\right)$ in luogo di $g\left(s^{2}\right)$, mostra appunto la presenza della forma $\mathscr{C}_{2}$ (in generale $\neq 0$ ) affetta dal coefficiente $s^{2} g_{0}=\int_{0}^{s^{2}} f d s^{2}$, il quale non può annullarsi per $s^{2}=r^{2}$ dovendo $f\left(s^{2}\right)$ soddisfare la (11). 
della $g_{1}\left(s^{2}\right)$. Tenendo conto della (26) si può dire soltanto che la $d\left[g_{1} \tilde{\theta}_{p-1}\right]$ si riduce sopra ogni $\Sigma_{p}$ ad una forma regolare anche nei punti di $\Delta_{n-p}$.

Si conclude dunque che, sia per l'una che per l'altra delle due strade tentate, non si può dare, almeno in generale; risposta immediata e afferma. tiva alla domanda del n. 10. Otterremo invece tale risposta e la effettiva costruzione delle forme elementari, soltanto dopo un esame approfondito della struttura delle forme $\tilde{\Omega}_{p}$ e $\tilde{\Theta}_{p-1}$ fornite dalle (18) e (22). $\grave{\mathrm{E}}$ opportuno però che prima stabiliamo alcune relazioni formali.

\section{Alcune relazioni formali.}

12. Indichiamo brevemente con $\sigma_{1}^{a b}$ la forma pfaffiana nelle variabili $z^{p+1}, \ldots, z^{n}:$

$$
\sigma_{1}^{a b}=\left\{\begin{array}{c}
a \\
b \beta
\end{array}\right\}_{0} d z^{\beta}
$$

dove, al solito, ̀े sott'inteso il segno di somma rispetto all' indice $\beta=p+1, \ldots, n$.

Avvertiamo che riserveremo le lettere latine $a, b, c, \ldots$ per gl' indici variabili da 1 a $p$, e le lettere greche $\alpha, \beta, \gamma, \ldots$ per gl' indici variabili da $p+1$ a $n$. Adotteremo inoltre sempre la convenzione di somma rispetto ad un indice alto ed uno basso, il che ci indurrà talora a spostare in basso l'indice delle coordinate $y^{a}$, scrivendo cioè indifferentemente $y_{a}$ in luogo di $y^{a}$.

La (14) si scrive allora:

$$
\delta y^{a}=d y^{a}+y_{b} \sigma_{1}^{a b}
$$

Tenuto conto ohe le linee coordinate $y^{a}$, nei punti di $\Delta_{n-p}$, sono ortogonali fra loro e a $\Delta_{n-p}$, risulta

$$
\left\{\begin{array}{c}
a \\
b \beta
\end{array}\right\}_{0}=\frac{1}{2}\left(\frac{\partial g_{a \beta}}{\partial y_{b}}-\frac{\partial g_{b \beta}}{\partial y_{a}}\right)
$$

dove i $g_{i j}$ sono i coefficienti della metrica di $M_{n}$ riferiti alle coordinate $\left(y^{a}, z^{\alpha}\right)$, e le loro derivate s'intendono calcolate nel punto $\left(0, z^{\alpha}\right)$ di $\Delta_{n-p}$. Ne seguono le relazioni di emisimmetria

e l'identità

$$
\sigma_{1}^{b a}=-\sigma_{1}^{a b}, \quad \sigma_{1}^{a a}=0
$$

$$
\delta s^{2}=2 y_{a} \delta y^{a}=2 y_{a} d y^{a}+2 y_{a} y_{b} \sigma_{1}^{a b}=d s^{2}+y_{a} y_{b}\left(\sigma_{1}^{a b}+\sigma_{1}^{b a}\right)=d s^{2},
$$

del resto a priori ovvia in base alle proprietà del parallelismo $W$ (n. 8), interpretando $s$ come misura di un vettore di componenti $y^{1}, \ldots, y^{p}$ applicato in un punto di $\Delta_{n-p}$.

Vediamo ora come si trasformino gli enti considerati per un cambiamento di coordinate del tipo espresso dalle (12). Ricordando l'ortogonalità del determinante delle $c^{a} \cdot a^{\prime}$, si ha $c^{a} \cdot a^{\prime}=c_{a}{ }^{a^{\prime}}$, dove con $c_{a}{ }^{a^{\prime}} \mathrm{s}^{\prime}$ indichi il reciproco 
dell' elemento $c^{a}{ }_{a^{\prime}}$. Tenuto conto di ciò si trovano senza difficoltà l' seguenti formule di trasformazione:

$$
\begin{aligned}
& d y^{a}=d \bar{y}^{a^{\prime}} c^{a} \cdot a^{\prime}+\bar{y}^{a^{\prime}} d c^{a} \cdot a^{\prime}, \\
& \sigma_{1}^{a b}=\bar{\sigma}_{1}^{a^{\prime} b^{\prime}} c^{a}{ }^{a}{ }^{\prime} c^{b}{ }^{b} \cdot b^{\prime}+c^{a}{ }_{\cdot{ }^{\prime}} d c_{b}{ }^{c^{\prime}}=\bar{\sigma}_{1}^{a^{\prime} b^{\prime}} c^{a} \cdot a^{\prime} c^{b} \cdot b^{\prime}-c^{b} \cdot{ }^{\prime} d c_{a}{ }^{c^{\prime}}, \\
& \delta y^{a}=\delta \bar{y}^{a^{\prime}} c^{a} \cdot a^{\prime} \text {. }
\end{aligned}
$$

La relazione (33) mostra che il differenziale $\delta y^{a}$ si comporta, per le trasformazioni (12), come un tensore contravariante rispetto all' indice $a$. Si può trarne subito una verifica formale dell' invarianza delle forme differenziali $\tilde{\Omega}_{p}, \tilde{\Theta}_{p-1}$ (invarianza già assicurata a priori, come abbiamo detto, dalla definizione stessa delle forme inviluppo; n. 9). Infatti basta scrivere le predette forme cosi

$$
\begin{aligned}
\tilde{\mathbf{\Omega}}_{p} & =\frac{1}{p !} \varepsilon_{a_{1} \ldots a_{p}} \delta y^{a_{1}} \times \ldots \times \delta y^{a_{p}}, \\
\tilde{\Theta}_{p-1} & =\frac{1}{p !} \varepsilon_{a_{2} \ldots a_{p}} y^{a_{1} \delta} y^{a_{2}} \times \ldots \times \delta y^{a_{p}},
\end{aligned}
$$

essendo $\varepsilon_{a_{1} \ldots a_{p}}$ il tensore numerico che vale $+10-1$ a seconda della parità o disparità della permutazione $a_{1}, \ldots, a_{p}\left({ }^{10}\right)$, e osservare che $\mathrm{i}$ prodotti esterni di tensori si comportano ancora come tensori.

Segnaliamo infine la forma differenziale di secondo grado nelle variabili $z^{p+1}, \ldots, z^{n}$ :

$$
\tau_{2}^{a b}=d \sigma_{1}^{a b}-\sum_{c} \sigma_{1}^{a c} \times \sigma_{1}^{b c}
$$

la quale si comporta anch' essa come un tensore rispetto agli indici $a, b\left({ }^{11}\right)$. Invero, differenziando la (32), si ottiene facilmente la formula di trasformazione:

Si ha inoltre:

$$
\tau_{2}^{a b}=\bar{\tau}_{2}^{\alpha^{\prime} b^{\prime}} e^{a} \cdot a^{\prime} c^{b} \cdot b^{\prime} \cdot
$$

$$
\tau_{2}^{a b}=-\tau_{2}^{b a}, \quad \tau_{2}^{a a}=0
$$

Forme elementari invarianti di grado $p=1$ e $p=2$.

13. Come abbjamo avvertito da principio (n. 1), ci limitiamo da questo punto in poi a considerare soltanto i primi quattro valori di $p$.

Il caso $p=1$ è ovvio e rientra del resto, come si è detto, nel caso semplice del n. 6. Tuttavia anche il procedimento generale conduce naturalmente allo stesso risultato. Invero, poichè per $p=1$ il parallelismo $W$ è integrabile (n. 8), risulta

$$
\tilde{\Omega}_{1}=\Omega_{1}=d y, \quad \tilde{\omega}_{1}=\omega_{1}=f\left(s^{2}\right) d y, \quad \tilde{\Theta}_{0}=\Theta_{0}=y
$$

(10) Il tensore $\varepsilon_{a_{1}} \ldots a_{p}$ si comporta qui come un tensore assoluto in quanto lo jacobiano della trasformazione (12), per quanto concerne 1 r $y^{a}$, vale +1 .

(11) Si vede subito che la forma $\tau_{2}{ }^{a b}$ ha per coefficienti i simboli di RIEManN relativi al parallelismo $W$. 
e, con qualunque scelta della funzione $g\left(s^{*}\right)$ tra gli integrali della (24) per $p=1$, si ottiene sempre $d\left[g \tilde{\Theta}_{0}\right]=f d y$, ciò̀ la stessa forma elementare che dà il n. 6 .

Passiamo al caso $p=2$. Tenato conto delle (18), (22), (30), si trova immediatamente

$$
\begin{gathered}
\tilde{\Theta}_{1}=\Theta_{1}+s^{z} \Phi_{1}, \\
\tilde{\Omega}=\Omega_{2}+d s^{2} \times \Phi_{1},
\end{gathered}
$$

ove $\Phi_{1}$ è la forma di $1^{\circ}$ grado regolare

$$
\Phi_{1}=-\frac{1}{2} \sigma_{1}^{12}
$$

La (36) può anche dedursi dalla (35) ricordando la (23) e la relazione analoga

$$
\tilde{\Omega}_{p}=\frac{p}{2} \frac{1}{s^{2}} \delta s^{2} \times \tilde{\Theta}_{x-1}=\frac{p}{2} \frac{1}{s^{2}} d s^{2} \times \tilde{\Theta}_{p-1}
$$

che si ottiene avuto riguardo all' identità $(31)$.

Differenziando la (35) abbiamo

$$
d \tilde{\Theta}_{1}=\tilde{Q}_{2}+s^{2} d \Phi_{1} .
$$

La precedente, tenuto conto dell' invarianza di $\tilde{\Theta}_{1}$ e di $\tilde{\Omega}_{2}$, mostra che anche la forma $d \Phi_{\mathrm{t}}$ è invariante. Ciò può del resto verificarsi direttamente, perchè dalla (37) risulta, ricordando la (34),

$$
d \Phi_{1}=-\frac{1}{2} d \sigma_{1}^{12}=-\frac{1}{2} \tau_{2}^{12}=-\frac{1}{4} \varepsilon_{a_{1} a_{2}} \tau_{2}^{a_{1} a_{2}}
$$

Dato il carattere tensoriale rilevato per la forma $\tau_{2}^{a_{1} a_{2}}$ (n. 12), si ha appunto l' invarianza della forma

$$
\wp_{2}=d \Phi_{1}=-\frac{1}{4} \varepsilon_{a_{1} a_{2}} \tau_{2} a_{1} a_{2}
$$

Possiamo dunque scrivere la (39) anche così

$$
d \tilde{\Theta}_{1}=\tilde{\Omega}_{2}+s^{2} \widetilde{G}_{2}
$$

e, se $g\left(s^{2}\right)$ ə̀ un integrale dell'equazione differenziale (24) per $p=2$, si ha poi

$$
\begin{aligned}
d\left[g \tilde{\Theta}_{1}\right] & =\left\{s^{2} g^{\prime}+g\right\} \tilde{\mathbf{\Omega}}_{z}+s^{2} g \sigma_{2} \\
& =f \tilde{\Omega}_{2}+s^{2} g \sigma_{2} .
\end{aligned}
$$

Si scelga ora come funzione $g\left(s^{2}\right)$ la $g_{1}\left(s^{2}\right)$ espressa dalla (28) per $p=2$; nonostante la singolarità di $g_{1}\left(s^{2}\right)$ in $s^{2}=0$, il coefficiente $s^{2} g_{1}\left(s^{2}\right)=\int_{r^{2}}^{s^{2}} f d s^{2}$, ohe appare nel secondo membro della (41), risulta regolare. Ne segue che la forma $d\left[g_{1} \tilde{\Theta}_{1}\right]$ è regolare di classe $v$ in tutto il tubo $T_{n}$, compresi $i$ punti del ciclo $\Delta_{n-2}$. 
In base al n. 11, la forma

$$
f\left(s^{2}\right) \tilde{\Omega}_{2}+\left[\int_{r^{\mu}}^{s^{2}} f\left(s^{2}\right) d s^{2}\right] \widetilde{G}_{2}
$$

costituisce perciò la desiderata forma elementare invariante di $2^{\circ}$ grado e di classe $v$ associata al ciclo $\Delta_{n-2}$.

Ha dunque risposta affermativa, per $p=2$, l'interrogativo del n. 10 . Vedremo che la risposta non è la stessa per $p>2$, benchè possa ugualmente giungersi alla costruzione di forme elementari.

Osservazione. - Una volta stabilita direttamente l'invarianza della forma $\widetilde{G}_{2}=d \Phi_{1}$, si sarebbe potuto ottenere la forma elementare costruita anche scrivendone a priori l'espressione invariante (42), quindi verificando per essa le condizioni $a$ ), b), c), d), del n. 5. Ciò che può farsi agevolmente osservando che la integrabilità della (42) risulta anche differenziando la (36). Osservazioni analoghe potrebbero farsi nel seguito.

Forme elementari invarianti di grado $p=3$.

14. Le relazioni che per $p=3$ tengono luogo delle (35), (36), sono:

$$
\begin{aligned}
& \tilde{\Theta}_{2}=\Theta_{2}+s^{2} \Phi_{2}+d s^{2} \times \Psi_{1}, \\
& \tilde{\Omega}_{3}=\mathbf{\Omega}_{3}+\frac{3}{2} d s^{2} \times \Phi_{2},
\end{aligned}
$$

essendo $\Phi_{2}, \Psi_{1}$ forme regolari dei gradi rispettivamente indicati.

La (44) si deduce subito dalla (43), avato riguardo alle relazioni generali (23) e (38). La deduzione della (43) non è invece così semplice come quella della sua analoga per $p=2$; per brevità ci limitiamo ad indicare soltanto le espressioni esplicite delle forme $\Phi_{2}$ e $\Psi_{1}$ :

$$
\begin{aligned}
& \Phi_{2}=-\frac{1}{6} \varepsilon_{a_{1} a_{2} a_{3}}\left[\sigma_{1}^{a_{1} a_{2}} \times d y^{a_{3}}+\frac{1}{2} \sigma_{1}^{a_{1} a_{2}} \times \sigma_{1}^{a_{2} b} y_{b}\right], \\
& \Psi_{1}=-\frac{1}{12} \varepsilon_{a_{1} a_{2} a_{3}} \sigma_{1}^{a_{1} a_{3}} y^{a_{3}} .
\end{aligned}
$$

Posto

$$
\Pi_{2}=\Phi_{2}+2 d \Psi_{1},
$$

si ha poi, ricordando la (34),

$$
\Pi_{2}=-\frac{1}{6} \varepsilon_{a_{1} a_{2} a_{3}} \tau_{2}^{a_{1} a_{2}} y^{a_{3}} .
$$


L'espressione (48), tenuto conto che la forma differenziale $\tau_{2}^{a_{1} a_{2}}$ e le coordinate $y^{\alpha_{3}}$ si trasformano con carattere tensoriale per $\mathrm{i}$ cambiamenti di variabili (n. 12), ci assicura che $\Pi_{2}$ è una forma invariante.

Procediamo ora analogamente al caso $p=2$. Dalla (43), differenziando e avendo riguardo alle (44), (47), abbiamo

$$
d \tilde{\Theta}_{2}=\tilde{\Omega}_{3}+s^{3} d\left[\frac{1}{s} \Pi_{2}\right]
$$

e, essendo $g\left(s^{2}\right)$ un integrale dell' equazione differenziale (24) per $p=3$,

$$
\begin{aligned}
d\left[g \tilde{\Theta}_{2}\right] & =\left\{\frac{2}{3} s^{2} g^{\prime}+g\left\{\tilde{\mathbf{\Omega}}_{3}+s^{3} g d\left[\frac{1}{s} \Pi_{2}\right]\right.\right. \\
& =f \tilde{\mathbf{\Omega}}_{3}+s^{3} g d\left[\frac{1}{s} \Pi_{2}\right] .
\end{aligned}
$$

Scelta come funzione $g\left(s^{2}\right)$ la $g_{1}\left(s^{2}\right)$ espressa dalla $(28)$ per $p=3$, il coefficiente $s^{3} g_{4}$ risulta regolare in $s^{2}=0$, ma non è ivi regolare la forma $d\left[\frac{1}{s} \Pi_{2}\right]$. Si constata dunque che il procedimento del n. 14 non conduce ora, almeno immediatamente, ad una forma elementare come per $p=2$.

Tuttavia si giunge lo stesso al fine desiderato se si considera il differenziale

$$
d\left[g \tilde{\Theta}_{2}-s^{2} g \Pi_{2}\right]=f \tilde{\Omega}_{3}-\frac{3}{2} f d s^{2} \times \Pi_{2} .
$$

Invero, tenuto conto dell' invarianza di $\Pi_{2}$ e delle proprietà della funzione $f\left(s^{2}\right)$, si conclude subito che la forma invariantivamente definita e regolare che appare a secondo membro della (49), costituisce effettivamente una forma elementare invariante di $3^{\circ}$ grado associata al ciclo $\Delta_{n-3}$.

Osserviamo che la forma elementare ora ottenuta non è l' unica forma elementare invariante costruibile, che si riduca sulle celle geodetiche $\Sigma_{3}$ alla forma $f \Omega_{3}=\omega_{3}$, dalla $q$ uale siamo partiti (n. 6). Infatti, se si aggiunge alla (49) un differenziale esatto invariante del tipo

$$
d\left[h\left(s^{2}\right) \Pi_{2}\right]=h^{\prime} d s^{2} \times \Pi_{2}+h d \Pi_{2},
$$

con $h\left(s^{2}\right)$ funzlone. regolare nell'intervallo $\left(0, r^{2}\right)$ annullantesi in $s^{2}=r^{2}$ insieme alle sue derivate fino all' ordine $v+1$, si ottiene ancora ovviamente una forma che soddisfa le condizioni dette. Posto $h^{\prime}\left(s^{2}\right)+\frac{3}{2} f\left(s^{2}\right)=f_{1}\left(s^{2}\right)$, si ha dunque la seguente generale forma elementare invariante di $3^{\circ}$ grado è di classe $v$ associata al ciclo $\Delta_{n-3}$;

$$
f \tilde{Q}_{3}+f_{1} d s^{2} \times \Pi_{2}+\left[\int_{r^{2}}^{s^{2}}\left(\frac{3}{2} f+f_{1}\right) d s^{2}\right] d \Pi_{3},
$$


dove $f_{t}\left(s^{2}\right)$ una funzione arbitraria di classe $v$ nell' intervallo $\left(0, r^{2}\right)$, soddisfacente alla condizione di annullarsi per $s^{*}=r^{*}$ insieme alle sue derivate fino all'ordine $v$.

Forme elementari invarianti di grado $p=4$.

15. Per $p=4$ si ottengono le relazioni seguenti, sulla cui deduzione non ei fermiamo $\left({ }^{12}\right)$ :

$$
\begin{aligned}
& \tilde{\Theta}_{3}=\Theta_{3}+s^{2} \Phi_{3}+d s^{2} \times \Psi_{2}, \\
& \tilde{\Omega_{4}}=\Omega_{4}+2 d s^{2} \times \Phi_{3},
\end{aligned}
$$

dove $\Phi_{3}, \Psi_{2}$ sono le forme regolari espresse dalle:

(53) $\Phi_{3}=-\frac{1}{16} \varepsilon_{a_{1} a_{2} a_{3} a_{4}}\left[\sigma_{1}^{a_{1} a_{9}} \times d\left(y^{a_{3}}, y^{a_{4}}\right)+\sigma_{1}^{a_{1} a} \times \sigma_{1}^{a_{3} b} y_{b} \times d y^{a_{4}}+\frac{1}{3} \sigma_{1}^{a_{1} a_{2}} \times \sigma_{1}^{a_{3} b} \times \sigma_{1}^{a_{4} c} y_{b} y_{c}\right]$,

$$
\Psi_{2}=-\frac{1}{16} \varepsilon_{a_{1} a_{2} a_{3} a_{4}}\left[\sigma_{1}^{a_{1} a_{2}} \times y^{a_{3}} d y^{a_{4}}-\frac{1}{2} \sigma_{1}^{a_{1} a_{2}} \times \sigma_{1}^{a_{b} b} y_{b} y^{a_{4}}\right]
$$

Si trova poi, per la forma

$$
\Pi_{3}=\Phi_{3}+d \Psi_{2},
$$

la decomposizione

$$
\Pi_{3}=\stackrel{\circ}{\Pi}_{3}+s^{2} \stackrel{*}{\Pi}_{3},
$$

dove :

$$
\begin{gathered}
\stackrel{\circ}{\Pi}_{3}=-\frac{1}{16} \varepsilon_{a_{1} a_{2} a_{i} a_{4}} \tau_{2}^{a_{1} a_{2}} \times y^{a_{3} \delta} y^{a_{4}}, \\
\stackrel{*}{\Pi}_{3}=-\frac{1}{64} \varepsilon_{a_{1} a_{2} a_{5} a_{4}}\left[\tau_{2}^{a_{2} a_{2}} \times \sigma_{1}^{a_{3} a_{4}}+\frac{1}{3} \sigma_{1}^{a_{1} a_{2}} \times \sum_{b} \sigma_{1}^{a_{3} b} \times \sigma_{1}^{a_{4} b}\right] .
\end{gathered}
$$

La (57) mostra senz'altro l' invarianza di $\stackrel{\circ}{\Pi}_{3}$. Non è lo stesso di III; perciò la forma $\mathrm{II}_{3}$ non è invariante, contrariamente a quanto avviene per la forma analoga $\Pi_{2}$ nel caso $p=3$ (n. 15). Ma, se si considera il differenziale di $\stackrel{*}{\Pi}_{3}$ si trova per esso la forma invariante:

$$
\widetilde{\sigma}_{4}=d \stackrel{*}{\Pi_{3}}=-\frac{1}{64} \varepsilon_{a_{1} a_{2} a_{3} a_{4}} \tau_{2}^{a_{3} a_{2}} \times \tau_{2}^{a_{3} a_{4}} .
$$

Differenziamo ora la (51). Avuto riguardo alle (52), (55), si ottiene:

$$
d \tilde{\Theta}_{3}=\tilde{\Omega}_{4}+s^{4} d\left[\frac{1}{s^{2}} \Pi_{3}\right] \text {. }
$$

(12) La (52) si deduce al solito immediatamente dalla (51) ricordando la (38); ma la deduzione delle $(51),(53)$, (5t) richiede sviluppi un po' complessi. 
Se poi $g_{1}\left(s^{2}\right)$ è al solito l'integrale dell'equazione differenziale (24) espresso dalla (28) per $p=4$, risulta, in base alle (38), (56), (59), (60):

$$
\begin{aligned}
d\left[g_{1} \tilde{\Theta}_{3}-s^{2} g_{1} \stackrel{\circ}{\Pi}_{3}\right] & =\left\{\frac{1}{2} s^{2} g_{1}^{\prime}+g_{1}\right\} \tilde{\Omega}_{4}-\left\{s^{2} g_{1}^{\prime}+2 g_{1}\right\} d s^{2} \times \stackrel{\circ}{\Pi}_{3}+g_{1} s^{1} d \Pi_{3}^{*} \\
& =f \tilde{\mathbf{S}}_{4}-2 f d s^{2} \times \stackrel{\circ}{\Pi}_{3}+2\left[\int_{r^{2}}^{s^{2}} f s^{2} d s^{2}\right] \widetilde{G}_{4} .
\end{aligned}
$$

Si è ottenuta così una forma invariante regolare nel tubo $T_{n}$ che costi* tuisce una forma elementare associata al ciclo $\Delta_{n-4}$. Ma, come già per $p=3$, tale forma non è unica. Se ne ottiene una più generale aggiungendo alla (61) il differenziale esatto invariante $d\left[h \Pi_{3}^{\circ}\right]$, dove la funzione $h\left(s^{2}\right)$ è dello stesso tipo considerato al n. 15. Posto qui: $h^{\prime}\left(s^{2}\right)-2 f\left(s^{2}\right)=f_{1}\left(s^{2}\right)$, si ha allora la seguente forma elementare invariante $d i 4^{\circ}$ grado e classe $v$ associata al ciclo $\Delta_{n-4}$ :

$$
f \tilde{\Omega}_{4}+f_{1} d s^{2} \times \stackrel{\circ}{\Pi}_{3}+\left[\int_{r^{2}}^{s^{2}}\left(2 f+f_{1}\right) d s^{2}\right] d \stackrel{\circ}{\Pi}_{3}+2\left[\int_{r^{2}}^{s^{2}} f s^{2} d s^{2}\right] \widetilde{\sigma}_{4}
$$

dove $f_{1}\left(s^{*}\right)$ è una funzione arbitraria soddisfacente alle stesse condizioni indicate alla fine del n. 15.

\section{Discussione del caso semplice.}

16. Abbiamo già detto (n. 6), cosa intendiamo per 《caso semplice» ed abbiamo mostrato che, quand' esso si verifica, il tubo $T_{n}$ costruito intorno a $\Delta_{n-p}$ può descriversi mediante le coordinate $\left(y^{a}, z^{x}\right)$, delle quali le $y^{a}$ hanno validità in grande, mentre le $z^{x}$ hanno validità locale.

Le equazioni $y^{a}=0(a=1, \ldots, p)$ rappresentano $\Delta_{n-p}$, e le $y^{a}=c^{a}$, essendo $c^{a}$ costanti abbastanza prossime a zero, un ciclo $\bar{\Delta}_{n-p}$ prossimo a $\Delta_{n \rightarrow p}$, che può pensarsi come deformato di $\Delta_{n-p}$. Due cicli $\bar{\Delta}_{n} p_{p}$ diversi non hanno aleun punto comune fra loro, nè con $\Delta_{n-p}$. Fissata una $\Sigma_{p}$ e un punto $\left(c^{1}, \ldots, c^{p}\right)$ su di essa, resta individuato un ciclo $\bar{\Delta}_{n-p}$ per il punto.

Possiamo dunque dire che, nel caso semplice, è soddisfatta la condizione seguente:

(S) Ogni ciclo $\Delta_{n-p}$ della base considerata in $M_{n}$ può deformarsi in $\infty^{p}$ modi, ed esiste qualche $p$-cella $\Sigma_{p}$ incontrante semplicemente $\Delta_{n-p}$ in un punto $P$, in guisa che per ogni punto $Q$ di $\Sigma_{p}$ passa un ciclo $\bar{\Delta}_{n-p}(Q)$ deformato, due cicli distinti essendo privi di punti comuni. Quando $Q$ tende a $P, \bar{\Delta}_{n-p}(Q)$ tende a $\Delta_{n-p}$.

Nella $(S)$ non v' è traccia della metrica introdotta in $M_{n}$. Ora, in una recente comunicazione [4], avevo già mostrato che, quando sia soddisfatta la 
candizione (S), può conseguirsi il teorema di CaRTaN-De RhaM in modo pressochè immediato e senza l' uso di una metrica su $M_{n}\left({ }^{13}\right)$.

Allorchè la varietà $M_{n}$ sia una superficie o una varietà 3-dimensionale, si può sempre soddisfare alla condizione $(S)$, giacchè è allora di necessità $n=1$ o $p=n-1$, casi che abbiamo già segnalati al n. 6 . Si vede anche subito che può soddisfarsi $(S)$ quando $M_{n}$ sia prodotto topologico di varietà sulle quali la $(S)$ sia già soddisfatta. Basta invero assumere come cicli fondamentali in $M_{n}$ quelli che, nell' inseguire il prodotto topologico, provengono dai cicli fondamentali delle varietà fattori del prodotto che soddisfanno ad $(S)$.

In [4] avevo anche osservato che la eondizione che $M_{n}$ sia orientabile ò necessaria perchè si possa soddisfare $(S)$. D'altronde l'orientabilità di $M_{n}$ è condizione essenziale per il teorema di CARTAN-DF RHAM che ci interessa. Ora si potrebbe pensare che l'orientabilità di $M_{n}$ fosse anche sufficiente per la validità di $(S)$. Ed anzi, io avevo espresso la presunzione intuitiva che cosi fosse.

Ma B. SEgre, in occasione della mia citata comunicazione, ha per il primo affacciato dubbi in proposito esponendo talune considerazioni tendenti a mostrare come improbabile l'anzidetta presunzione. In seguito, grazie ad una semplice osservazione di G. ZAPPA, ho potuto dare alcuni esempi in contrario, che passo ad esporre.

17. L'osservazione di G. ZAPPA è questa. Sopra una superficie algebrica, che, considerata dal punto di vista topologico, è una varietà 4-dimensionale orientabile, esistono 2-cicli orientabili i quali hanno un indice di KRONECKER d'intersezione con se stessi diverso da zero. Sono tali p. es. i 2-cicli che rappresentano le curve algebriche sezioni piane della superficie (pensata nello spazio ordinario) in quanto l'indice di KRoneoKer relativo uguaglia, com'è noto, l'ordine della superficie. Uno di questi cicli, $\Delta_{2}$, non è perciò suscettibile di venir deformato in un ciclo, $\bar{\Delta}_{2}$, privo di intersezioni con $\Delta_{2}$, chè altrimenti sarebbe $\left(\Delta_{2}, \Delta_{2}\right)=\left(\Delta_{2}, \bar{\Delta}_{2}\right)=0$.

Se ne traggono immediatamente gli esempi che c'interessano. Il più semplice è già offerto dalla riemanniana del piano proiettivo complesso. Infatti su tale varietà non ' '’è che un sol ciclo 2-dimensionale indipendente, $\Delta_{2}$, che ̀̀ omologo ad una retta del piano, ond'è $\left(\Delta_{2}, \Delta_{2}\right)=1$, comunque si scelga il ciclo base $\Delta_{2}$.

Lo stesso ragionamento è valido per la riemanniana $M_{4 m}$ di uno spazio proiettivo complesso $S_{2 m}$, nei riguardi della base $2 m$-dimensionale che è costituita da un sol ciclo $\Delta_{2 m}$ omologo all'immagine di un $S_{m}$ di $S_{z m}$.

(13) Il ragionamento allora fatto è sostanzialmente lo stesso di quello del n. 6, Oss. $2^{a}$. La metrica di $M_{n}$ non ha invero alcuna funzione essenziale in questo caso. Se ne prescinde subito costruendo innanzi tutto un tubo $T_{n}$ intorno a $\Delta_{\hat{n}-p}$ come luogo dei cicli $\bar{\Delta}_{n-p}(Q)$ al variare di $Q$ sulla cella $\Sigma_{p}$. Se poi $y^{1}, \ldots, y^{p}$ sono coordinate qualunque in $\Sigma_{p}$, un punto variabile in $T_{n}$ può individuarsi mediante le coordinate $\left(y^{a}, z \alpha\right)$, delle quali le $z \alpha$ hanno al solito validità locale. II ragionamento prosegue poi come nel passo citato del n. 6 . 
Se si considera invece una superficie quadrica complessa la cui riemanniana $M_{4}$, com'è ben noto, ¿ equivalente al prodotto topologico di due supercie sferiche reali $\Sigma^{\prime}, \Sigma^{\prime \prime}$, si vede che può assumersi in $M_{4}$ una base 2-dimensionale costituita sia da cicli che soddisfanno la condizione $(S)$, sia da cicli che non la soddisfanno.

Una base del primo tipo è data dai 2-cicli $\Delta^{1}=\Sigma^{\prime} \times P^{\prime \prime}, \Delta^{2}=P^{\prime} \times \Sigma^{\prime \prime}$ essendo $P^{\prime}, p^{\prime \prime}$ punti di $\Sigma^{\prime}, \Sigma^{\prime \prime}$. Invero, basta per soddisfare $(S)$ assumere come cicli deformati di $\Delta^{4}, \Delta^{2}$, i cicli $\bar{\Delta}^{t}=\Sigma^{\prime} \times \bar{P}^{\prime \prime}, \bar{\Delta}^{2}=\bar{P}^{\prime} \times \Sigma^{\prime \prime}$, dove $\bar{P}^{\prime}, \bar{P}^{\prime \prime}$ siano punti degli intorni di $P^{\prime}, P^{\prime}$ su $\Sigma^{\prime}, \Sigma^{\prime \prime}$.

Una base del secondo tipo è invece quella costituita dal cicli $\Delta^{1}, \Delta^{1}+\Delta^{2}$. Infatti, con convenienti orientazioni di $\Sigma^{\prime}, \Sigma^{\prime \prime}, \Sigma^{\prime} \times \Sigma^{\prime \prime}$, si ha immediatamente

$$
\left(\Delta^{1}, \Delta^{1}\right)=\left(\Delta^{2}, \Delta^{2}\right)=0, \quad\left(\Delta^{1}, \Delta^{2}\right)=\left(\Delta^{2}, \Delta^{1}\right)=1 .
$$

Il ciclo $\Delta^{1}+\Delta^{2}$ non soddisfa allora $(S)$, poichè, tenuto conto delle precedenti, risulta

$$
\left(\Delta^{1}+\Delta^{2}, \Delta^{1}+\Delta^{2}\right)=2\left(\Delta^{1}, \Delta^{2}\right)=2 .
$$

Non v’è difficoltà a sostituire, se si vuole, il ciclo riducibile $\Delta^{1}+\Delta^{2}$ con un ciclo irriducibile, p. es. l'immagine di una conica della quadrica rappresentata da $\Sigma^{\prime} \times \Sigma^{\prime \prime}$.

\section{Forme invarianti di Wirtinger.}

18. Sia $S_{m}\left(\xi^{0}, \xi^{1}, \ldots, \xi^{m}\right)$ lo spazio proiettivo complesso $m$-dimensionale, e $\xi^{k}$ coordinate omogenee in esso. Ricordiamo che entro la varietà reale $M_{2}$ immagine di $S_{m}$, pad̀ introdursi (Mannoury, Fubini, Study) una metrica riemanniana invariante rispetto al gruppo delle omografie di $S_{m}$ in sè che lascia fissa la forma quadratica hermitiana

$$
H=\xi^{0} \bar{\xi}^{0}+\xi^{1} \bar{\xi}^{1}+\ldots+\xi^{m} \bar{\xi}^{m},
$$

essendo $\bar{\xi}^{k}$ il complesso coniugato di $\xi^{k}$.

Tale metrica può scriversi nel modo semplice, indicato da KAEHLER,

$$
d s^{2}=4 \frac{\partial^{2} \log H}{\partial \xi^{h} \partial \bar{\xi}^{k}} d \xi^{n} d \bar{\xi}^{n}
$$

con la solita convenzione di summa.

Siccome i numeri di BeTri di dimensione dispari della varietà $M_{2 n x}$ sono tutti nulli e quelli di dimensione pari valgano 1 (VAN DER WAERDEN), non v'è da considerare in $M_{2 m}$ altro che una forma differenziale indipendente che risolve il problema esistenziale di CARTAN-DE RHAM per ogni valore pari del grado $p$. E noto che si possono assumere come forme risolventi le forme invarianti considerate da WIRTINGER [9]:

$\omega_{2}=2 i \frac{\partial^{2} \log H}{\partial \xi^{h} \partial \bar{\xi}^{k}} d\left(\xi^{h}, \bar{\xi}^{k}\right), \omega_{4}=\frac{1}{2 !} \omega_{2} \times \omega_{2}=\frac{1}{2 !}\left(\omega_{2}\right)^{2}, \ldots, \omega_{2(m-1)}=\frac{1}{(m-1) !}\left(\omega_{2}\right)^{m-1}$. 
Come abbiamo detto al n. 1, riteniamo che le forme di Wirtinger rien. trino nella classe delle forme elementari invarianti costruite nelle pagine precedenti (per $p \leq 4$ ). In realtà abbiamo per il momento verificato questa affermazione soltanto per $p=2, m=2$ e $p=4, m=6$. Ci limitiamo percio ad accennarne molto brevemente.

Posto $p=2 q$, come unico eiclo base del gruppo di Berrat di dimensione $2 m-2 q$ in $M_{2 m}$, si assuma il ciclo $\Delta_{2 m-2 q}=M_{2 m-2 q}$ immagine di uno spazio lineare $S_{m-q}$ di $S_{m}$. Si dimostra allora, innanzi tutto, che le varietà geodetiche ortogonali a $\Delta_{2 m-2 q}$ sono le immagini $M_{2 q}$ degli $S_{q}$ di una stella che ha per sostegno un $S_{q-1}$ sghembo con il fissato $S_{m-q}$. La cella geodetica $\Sigma_{2 q}(P)$ con centro in un punto $P$ di $\Delta_{2 m-2 q}$ risulta perciò contenuta in una tale $M_{2 q}$. Se ora si assume il raggio $r$ delle $\Sigma_{2 q}$ uguale alla massima distanza. $\pi$, di due punti di $M_{2 m}$, ogni $\Sigma_{2 q}$ viene ad esaurire la corrispon. dente $M_{2 q}$, e il tubo $T_{2 m}$ descritto dalle $\Sigma_{2 q}(P)$ al variare di $P$ su $\Delta_{2 m-2 q}$ esaurisce l'intera varietà $\mathrm{M}_{2 m}$. Il contorno di $T_{2 m}$ si ripiega su se stesso e si abbassa di dimensione, riducendosi alla varietà $M_{2 q-2}$ immagine del predetto $S_{q-1}$ e perdendo cosi il carattere di frontiera.

Con una conveniente scelta delle funzioni arbitrarie che entrano nella espressione della forma elementare invariante associata a $\Delta_{z m-2 q}$ e definita nel tubo $T_{2 m}=M_{2 m}$, si ottiene appunto la forma di Wintunger $\omega_{2 q}$ (a meno di una inessenziale costante moltiplicativa dipendente dalla condizione vincolativa $d$ ), che si è assunta nella definizione di forma elementare; n. 5).

\section{BIBLIOGRAFIA}

[1] E. Cartan, «Comptes rendus », 187 (1928), pag. 196.

[2] W. V. D. Hodge, The Theory and Appl. of Harmonic Integrals, London (1941).

[B] S. Lefschetz, Topology, New York (1980).

[4] E. Martinklu, Comunicazione al III Congresso U.M.I. in Pisa (1948), riprodota in "Bollettino U.M.I. ", s. $3^{a}, 4$ (1949).

[5] G. De Rhaм, "Journal Math. pures et appl, „, 10 (1931), pag. 115.

[6] F. Severi, Lezioni di analisi, Bologna (1942), Vol. II.

[7] H. WEYL, "Math. Zeitsehrift *, 12 (1922), pag. 154.

[8] H. Whitney, "Annals of Math. ", 37 (1936), pag. 645.

[9] W. Wirtinger, "Monatshefte für Math. u. Phys. », 44 (1986), pag. 343. 